\title{
Mycobacterium tuberculosis Complex Lineage 3 as Causative Agent of Pulmonary Tuberculosis, Eastern Sudan ${ }^{1}$
}

Yassir A. Shuaib, Eltahir A.G. Khalil, Lothar H. Wieler, Ulrich E. Schaible, Mohammed A. Bakheit, Saad E. Mohamed-Noor, Mohamed A. Abdalla, Glennah Kerubo, Sönke Andres, Doris Hillemann, Elvira Richter, Katharina Kranzer, Stefan Niemann, ${ }^{2}$ Matthias Merker ${ }^{2}$

Pathogen-based factors associated with tuberculosis (TB) in eastern Sudan are not well defined. We investigated genetic diversity, drug resistance, and possible transmission clusters of Mycobacterium tuberculosis complex (MTBC) strains by using a genomic epidemiology approach. We collected 383 sputum specimens at 3 hospitals in 2014 and 2016 from patients with symptoms suggestive of TB; of these, 171 grew MTBC strains. Whole-genome sequencing could be performed on 166 MTBC strains; phylogenetic classification revealed that most $(73.4 \% ; n=122)$ belonged to lineage $3(L 3)$. Genome-based cluster analysis showed that 76 strains $(45.9 \%)$ were grouped into 29 molecular clusters, comprising 2-8 strains/patients. Of the strains investigated, 9.0\% (15/166) were multidrug resistant (MDR); $10 \mathrm{MDR}$ MTBC strains were linked to 1 large MDR transmission network. Our findings indicate that $\mathrm{L} 3$ strains are the main causative agent of TB in eastern Sudan; MDR TB is caused mainly by transmission of MDR L3 strains.

Tuberculosis (TB) remains a major global health 1 problem; 10 million new cases were reported in 2018 (1). In Sudan, the estimated national TB incidence in 2018 was 71/100,000 persons; a total of

Author affiliations: Freie Universität Berlin, Berlin, Germany (Y.A. Shuaib, L.H. Wieler); Research Center Borstel, Borstel, Germany (Y.A. Shuaib, U.E. Schaible, S. Andres, D. Hillemann, S. Niemann, M. Merker); Sudan University of Science and Technology, Khartoum, Sudan (Y.A. Shuaib, S.E. MohamedNoor, M.A. Abdalla); University of Khartoum, Khartoum (E.A.G. Khalil, M.A. Bakheit); Robert Koch Institute, Berlin (L.H. Wieler); Kenyatta University, Nairobi, Kenya (G. Kerubo); Labor Limbach, Heidelberg, Germany (E. Richter); London School of Hygiene and Tropical Medicine, London, UK (K. Kranzer); German Center for Infection Research, Borstel Site, Borstel (S. Niemann, M. Merker)

DOI: https://doi.org/10.3201/eid2603.191145
20,638 cases were reported (1). However, the TB burden is by no means homogeneous across the country. For instance, in eastern Sudan, TB notifications reached 275/100,000 persons in 2012 (2,3). Prevalence of multidrug-resistant TB (MDR TB) (i.e., resistant to isoniazid and rifampin) was estimated at $2.9 \%$ in new and $13 \%$ in retreatment cases; however, studies have reported MDR TB rates of $6 \%-22 \%(1,4-10)$.

Ongoing transmission is one of the key challenges for TB control programs, especially in countries with a high TB burden $(1,11)$. In recent years, molecular techniques have been increasingly used to clarify and trace transmission of Mycobacterium tuberculosis complex (MTBC) strains and to direct and guide targeted TB control actions $(12,13)$. However, availability of molecular techniques is limited in many countries in Africa with a high TB burden (11).

In Sudan, drug-resistant TB often goes undetected, resulting in inadequate treatment, illness, death, and ongoing transmission $(1,14)$. Local laboratories have limited access to mycobacterial culture and drug susceptibility testing (DST) or DNA-based techniques (14). Therefore, MDR TB rates might be underestimated in eastern Sudan. In addition, mutations that mediate drug resistance have not been investigated.

Taken together, these factors indicate that, although TB is a huge health problem in eastern Sudan, precise data on the phylogeny and transmission dynamics of MTBC strains, as well as on resistance patterns, is sparsely available $(2,3,7,8,15)$. Studies using molecular epidemiologic tools are rare and have used classical genotyping techniques, such as

${ }^{1}$ Preliminary results from this study were presented at the 39th Annual Congress of the European Society of Mycobacteriology, July 1-4, 2018, Dresden, Germany.

${ }^{2}$ These authors contributed equally to this article. 
spoligotyping, which cannot deduce direct transmission events $(5,15)$. New techniques, such as wholegenome sequencing (WGS), offer the highest resolution for MTBC genotyping and provide precise information on resistance mutations $(16,17)$. We applied state-of-the-art phenotypic and molecular assays to investigate specimens collected from patients with symptoms suggestive of pulmonary TB, including new and retreatment cases, to analyze the MTBC population structure, putative transmission events, and DST profiles in eastern Sudan.

\section{Methods}

\section{Study Design and Setting}

We recruited patients with symptoms suggestive of pulmonary $\mathrm{TB}$ who had positive sputum smears and agreed to participate in this cross-sectional study. Patients had been treated in the outpatient departments at public hospitals in Kassala, Port Sudan, and El-Gadarif in eastern Sudan over 2 recruitment periods, June-October 2014 and January-July 2016. We collected spot and early morning sputum samples. If 1 sample was smear positive, the 2 samples were pooled and stored for $\leq 6$ months at $-20^{\circ} \mathrm{C}$. Shortly before we shipped each sample to the National Reference Center (NRC) for Mycobacteria, Borstel, Germany, we transferred a volume of $\leq 2 \mathrm{~mL}$ to a screwcapped Eppendorf tube; the samples were shipped in 2 separate batches.

\section{Mycobacterial Culture and Identification}

Sample decontamination, smear microscopy, and mycobacterial culture were performed at the NRC $(18,19)$. We extracted DNA using a QIAamp DNA Mini Kit 250 (QIAGEN, https://www.qiagen.com) according to the instructions of the manufacturer for quantitative PCR (qPCR). We extracted DNA by the boiling/sonication method for conducting line probe assays (LPAs) such as GenoType Mycobacterium CM and GenoType Mycobacterium MTBC (Hain Lifescience, https://www.hain-lifescience.de) (19). We used cetyl trimethylammonium bromide for DNA extraction for WGS (20). We transferred the extracted DNA to new Eppendorf tubes and stored it at $-20^{\circ} \mathrm{C}$ until used.

We used an in-house qPCR detecting MTBC and nontuberculous mycobacteria (NTM) DNA to test available culture-negative/contaminated samples (21). We ran the qPCR experiments using the RotorGene 2000 (Corbett Research Pty Ltd, http://www. australianexporters.net). We used LPAs (Hain Lifescience, https://www.hain-lifescience.de) according to the manufacturer's instructions to classify isolated mycobacteria into MTBC or NTM and to differentiate the MTBC species.

We identified NTM species using $16 S$ rRNA, internal transcribed spacer (ITS) DNA fragment sequencing, or both (22). We sequenced the complete PCR products on an automated DNA sequencer (ABI 377; Applied Biosystems, https://www.thermofisher.com) by cycle sequencing using the Big Dye RR Terminator Cycle Sequencing Kit (Applied Biosystems). We aligned the resulting sequences and compared them with the sequences of the International Nucleotide Sequence Database Collaboration.

\section{Drug Susceptibility Testing}

We performed phenotypic DST (pDST) for resistance to streptomycin $1 \mu \mathrm{g} / \mathrm{mL}$, isoniazid $0.1 \mu \mathrm{g} /$ $\mathrm{mL}$, rifampin $1 \mu \mathrm{g} / \mathrm{mL}$, ethambutol $5 \mu \mathrm{g} / \mathrm{mL}$, and pyrazinamide $100 \mu \mathrm{g} / \mathrm{mL}$ for all MTBC isolates (18). We further investigated isolates with resistance to $\geq 1$ first-line drug for resistance to ofloxacin $2 \mu \mathrm{g} / \mathrm{mL}$, amikacin $1 \mu \mathrm{g} / \mathrm{mL}$, and capreomycin $2.5 \mu \mathrm{g} / \mathrm{mL}$ (23).

We further evaluated strains that had a mutation in the $e m b B$ codons 306, 406, or 497 (as detected by WGS) but tested phenotypically susceptible to ethambutol at the critical concentration by determining ethambutol MICs. We assessed concentrations of $1.25,2.5,3.75$, and $5.0 \mu \mathrm{g} / \mathrm{mL}$ in the BACTEC MGIT 960 system (Becton Dickinson, https:/ / www.bd.com) $(24,25)$.

\section{WGS}

We performed WGS using the Illumina Nextera (XT) kit (https://www.illumina.com) (26). We sequenced isolates with a minimum average genome coverage of $50 \times$. We used single-nucleotide polymorphisms (SNPs) occurring in $\geq 4$ forward and $\geq 4$ reverse reads, 4 reads calling the allele with a Phred score $\geq 20$, and a minimum variant frequency of $75 \%$ for a concatenated sequence alignment (27). In the comparative genomic analysis, we allowed 5\% of all samples to miss these coverage and frequency thresholds at individual positions and called the majority allele $(>50 \%$ variant frequency) to not lose sequence information in genome regions with lower average coverage. We excluded repetitive region and drug resistance associated genes for phylogenetic reconstruction.

\section{Phylogenetic Inference}

We calculated a maximum-likelihood tree with FastTree using the concatenated sequence alignment and a general time-reversible substitution model (28). We conducted inspection and rooting of the 
maximum likelihood tree using FigTree software and performed the graphical presentation using the online tool EvolView (29). We calculated maximum parsimony trees with BioNumerics version 7.6 (Applied Maths, https://www.applied-maths.com) using the concatenated sequence alignment (30).

\section{Molecular Drug Resistance Prediction}

We screened the $r p s L$, $r r s$, and gidB genes for mutations that confer resistance to streptomycin and the kat $G$ and inhA genes and the fabG1-inhA promoter for resistance to isoniazid (31). We inferred rifampin resistance by mutations in the $r p o B$ gene. Moreover, we also noted putative compensatory mutations in the rpoA and rpoC genes (for rifampin resistance) and the $a h p C$ gene (for isoniazid resistance). We investigated the $e m b A$, $e m b B$, and $e m b C$ genes for resistance conferring mutations to ethambutol and screened the $p n c A$ gene for mutations associated with resistance to pyrazinamide (31). We investigated the gyrA and gyrB genes for resistance to fluoroquinolones and investigated the $r r s$ gene for resistances against kanamycin, amikacin, and capreomycin. In addition, we screened the eis promoter region for resistance against KAN and the tlyA for resistance against capreomycin. For ethionamide, we investigated the eth $A$ and inh $A$ genes and the fabG1-inhA promoter and for para-aminosalicylic acid, we investigated the ribD, thy $A$, thy $X$, and folC genes (31).

\section{Statistics}

We used SPSS version 20.0 (https://www.ibm.com) for all appropriate statistical analyses. We obtained descriptive statistics of the variables, including frequencies and proportions. We analyzed differences between groups by using the $\chi^{2}$ or Fisher exact test; $\mathrm{p} \leq 0.05$ denoted statistical significance (32).

\section{Ethics Considerations}

Scientific and ethics approval for the study was provided by the National Research Ethics Committee, Federal Ministry of Health, Khartoum, Sudan, and by the Institutional Review Board of the Institute of Endemic Diseases, University of Khartoum, Khartoum, Sudan (no. 85-03-09). We obtained written informed consent for participation in the study from participants or, in case of children or illiterate patients, their guardians.

\section{Results}

\section{Study Population}

Sputum samples were provided by smear-positive patients with TB from 3 areas in eastern Sudan in $2014(\mathrm{n}=101)$ and $2016(\mathrm{n}=282)$ (Figure 1). Based on hospital records, we included $10 \%-20 \%$ of all patients who received diagnoses of TB during the study period. We collected patient-derived samples from 161 patients (42\%) in El-Gadarif, 133 patients $(34.7 \%)$ in Kassala, and 89 patients (23.3\%) in Port Sudan hospitals. Patients who provided samples had a median age of 35 years (interquartile range 25-45 years); most $(245 / 383 ; 66 \%)$ were male. In addition, $81.5 \%$ $(312 / 383)$ were new and $5.5 \%(21 / 383)$ were retreatment TB cases; data on TB treatment history were unavailable for $13.0 \%$ (50/383) (Table 1). Comparison of the 2 patient cohorts revealed no significant difference between the proportions of L3 strains ( $p=0.068$ by Fisher exact test) but the 2014 cohort contained more drug-resistant ( $p=0.019$ by Fisher exact test) and clustered ( $p=0.016$ by Fisher exact test) strains (Table 2).

\section{Mycobacterium Isolation and Species Identification}

Of all collected specimens, 51.2\% (196/383) were culture positive for mycobacteria; LPAs identified most ( $\mathrm{n}=171,87.2 \%$ ) as MTBC (Figure 1$)$. The rest of the specimens were either culture negative or contaminated; we tested them with qPCR and Sanger sequencing for mycobacterial DNA detection and species identification (Figure 1) (14).

MTBC Population Structure and Genome-Based Clusters We performed WGS successfully on 166 MTBC strains. We built a maximum-likelihood phylogeny upon a concatenated sequence alignment comprising 11,932 SNPs to investigate the MTBC population structure (Figure 2). We performed MTBC (sub)lineage (L) classification with an SNP bar code nomenclature that was recently introduced (33). Strains of L3 (Delhi/CAS) were predominant (73.5\%, 122/166), followed by L4 (Euro-American) strains (23.5\%, 39/166). We further classified L4 strains into several sublineages (Appendix 1 Figure 1, https://wwwnc. cdc.gov/EID/article/26/3/19-1145-App1.pdf). The remaining isolates belonged to L1 $(2.4 \%, 4 / 166)$ and L2 $(0.6 \%, 1 / 166)$.

To obtain an indication about putative recent transmission events, we conducted a cluster analysis based on a pairwise SNP distance of $\leq 12$ SNPs between any 2 strains $(12,34)$. Overall, $45.9 \%$ (76/166) of the strains were grouped in 29 clusters comprising 2-8 isolates/patients (Appendix 1 Figure 2). L3 strains were observed with a higher clustering rate $(52.5 \%, 64 / 122)$ than L4 isolates $(28.2 \%, 11 / 39)(\mathrm{p}=$ 0.016 by Fisher exact test). Two of the 4 L1 strains were clustered; no L2 strains were assigned to a WGS 


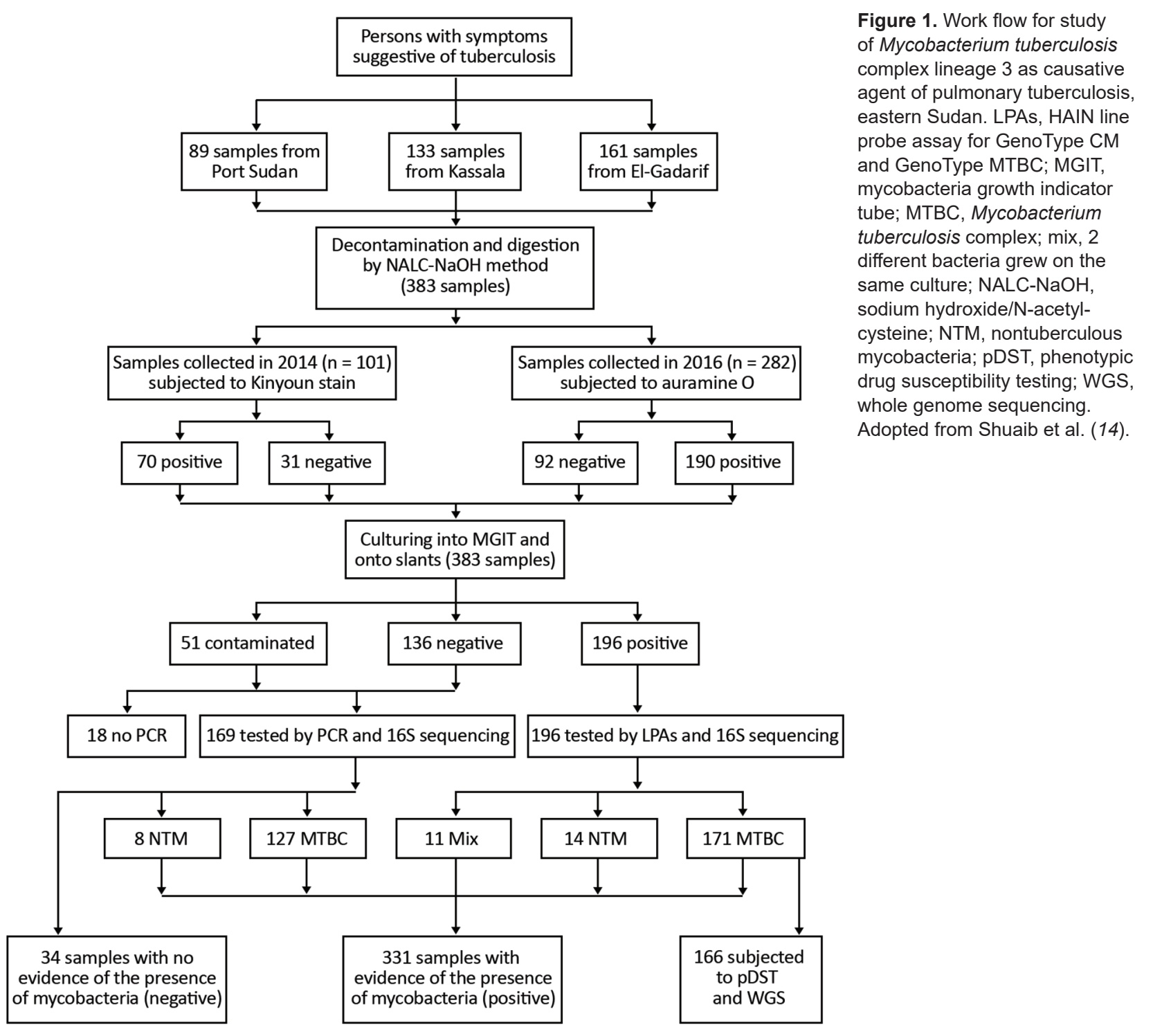

cluster. Moreover, considering a stricter threshold $(\leq 5$ SNPs), 36.7\% (61/166) isolates were still connected in 24 clusters comprising 2-8 isolates/patients (Appendix 1 Figure 3).

\section{pDST and Genotypic DST}

To determine resistance levels and related genomic variants, we performed $\mathrm{pDST}$ and genomic resistance predictions or genotypic DST (gDST) and compiled detailed data on resistances and resistance conferring mutations (Tables 3, 4; Appendix 1 Table). Overall, $21.7 \%(36 / 166)$ of the strains showed resistance to $\geq 1$ of the tested first-line antimicrobial drugs by pDST, including 15 (9.0\%) MDR and 21 (12.7\%) nonMDR strains (Appendix 2, https:/ / wwwnc.cdc.gov/ EID/article/26/3/19-1145-App2.xlsx). Strikingly, all MDR and $76.1 \%$ of non-MDR strains belonged to L3.
Furthermore, beyond the MDR classification, we found that L3 strains in eastern Sudan were more often found with drug resistances as compared with L4 strains (L3, 31/122, 25.4\%; L4, 4/39, 10.3\%; $p=0.048$ by Fisher exact test).

We detected resistance to streptomycin in 19.9\% $(33 / 166)$ of the strains, mediated by mutations in rspL (Lys43Arg, Lys88Arg, and Lys88Met), gidB (e.g., Ala138Val), and rrs (514, a/c) genes. We observed all isoniazid-resistant strains $(10.2 \%, 17 / 166)$ either with a mutation in katG (Ser315Thr and Ser315Asn) that changes catalase-peroxidase activities or in the promoter region of the drug target InhA, fabG1-inhA $(-15 \mathrm{c} / \mathrm{t})$, which also confers resistance to the secondline drug ETH. Resistance to rifampin was found in $10.2 \%(17 / 166)$ of the strains and was mediated by mutations in the rpoB gene (Ser450Leu, His445Tyr, 
His445Asn, and His445Asp). We found 1 ethambutol-resistant strain $(0.6 \%)$ with the mutation $\mathrm{embB}$ Gln497Arg. However, we also detected 11 additional mutations associated with ethambutol resistance in the embB gene (10 Met306Ile and 1 Met306Val) but with MICs ranging from 1.25 to $5 \mu \mathrm{g} / \mathrm{mL}$, classifying these strains as phenotypically susceptible based on the recommended critical concentration for ethambutol. With regard to pyrazinamide, we identified 1 strain $(0.6 \%)$ with the mutation pncA Gln10Arg, coinciding with phenotypic pyrazinamide resistance.

A detailed comparison of the pDST and gDST results revealed a high sensitivity and specificity for isoniazid, rifampin, and pyrazinamide resistance prediction by WGS (Table 4). For ethambutol, we determined high-confidence resistance SNPs at codon 306, 406, or 497; however, varying levels of ethambutol MICs in the strains with mutations resulted in a very low positive predictive value. For streptomycin, we considered the gidB mutations (Phe12Ser, Arg39Pro, Trp45STP, Ser136STP, Iso114Ser, and deletions at positions 4408101, 4408017, and 4408116) to be mutations with an unclear effect. However, these strains eventually tested phenotypically resistant to streptomycin, leading to a reduced sensitivity.

All strains with resistances to $\geq 1$ first-line antimicrobial drug were phenotypically and genotypically susceptible to ofloxacin, capreomycin, and amikacin. We identified no genotypic resistance marker mediating para-aminosalicylic acid resistance.

Based on a 12-bp SNP threshold between any 2 strains, $80.0 \%(12 / 15)$ of the MDR strains were clustered or connected (i.e., associated with recent transmission); based on $\leq 5$ SNPs distance, $60 \%$ (9/15) of the MDR strains were clustered (Appendix 1 Figure 4 , panel A). Most of the clustered strains at $\leq 12$ SNPs were isolated from patients in Kassala and grouped in clusters 4 and 29. These strains also shared the same rpsL (Lys43Arg) and the katG (Ser315Thr) mutations but harbored different mutations in the rpoB gene; strains of cluster 4 had the Ser450Leu mutation,
Table 1. Demographic characteristics of tuberculosis patients investigated in eastern Sudan

\begin{tabular}{lcc}
\hline Characteristic & $\begin{array}{c}\text { No. recruited } \\
\text { patients }\end{array}$ & $\begin{array}{c}\text { \% Recruited } \\
\text { patients }\end{array}$ \\
\hline Origin & & \\
Kassala & 133 & 34.7 \\
El-Gadarif & 161 & 42.0 \\
Port Sudan & 89 & 23.3 \\
\hline Sex & & \\
M & 245 & 64.0 \\
F & 123 & 32.1 \\
Not available & 15 & 3.9 \\
\hline Age, y & & \\
$<25$ & 76 & 19.8 \\
$25-40$ & 170 & 44.4 \\
$>40$ & 108 & 28.2 \\
Not available & 29 & 7.6 \\
\hline Treatment history & & \\
Retreatment & 21 & 5.5 \\
New & 312 & 81.5 \\
Not available & 50 & 13.0 \\
\hline All patients & 383 & 100 \\
\hline
\end{tabular}

whereas strains of cluster 29 exhibited a His445Tyr mutation. This finding points toward a close relationship between the strains of both clusters that likely emerged from a common recent ancestor already being polyresistant to streptomycin and isoniazid (Appendix 1 Figure 4, panel B). Furthermore, all 5 strains of cluster 29 had the $e m b B$ Met306Ile mutation, but 1 of them also had the mutation embB Gly406Asp. Within cluster 4, two strains acquired the mutation $e m b B$ Met306Ile independently, and 1 acquired $e m b B$ Met306Val, as judged by the tree topology (Appendix 1 Figure 4, panel B). Moreover, among all drugresistant strains, only 1 strain was identified with resistance to pyrazinamide mediated by the mutation pncA Gln10Arg.

\section{Discussion}

By using conventional diagnostics and WGS, we showed that pulmonary TB in eastern Sudan is caused predominantly by L3 strains (Delhi/CAS). Drug resistance and recent transmission were associated with L3 strains, accentuating the key role of L3 strains in TB epidemiology in eastern Sudan. In addition, most

\begin{tabular}{|c|c|c|c|c|}
\hline \multirow[b]{2}{*}{ Variable } & \multicolumn{2}{|c|}{ Year of sample collection, no. (\%) isolates } & \multirow[b]{2}{*}{ Total no. } & \multirow[b]{2}{*}{$p$ value } \\
\hline & 2014 & 2016 & & \\
\hline Lineage & & & & 0.068 \\
\hline L3 & $51(82.3)$ & $71(68.3)$ & 122 & \\
\hline Non-L3 & $11(17.7)$ & $33(31.7)$ & 44 & \\
\hline DST & & & & 0.019 \\
\hline Any drug resistance & $20(32.3)$ & $16(15.4)$ & 36 & \\
\hline No drug resistance & $42(67.7)$ & $88(84.6)$ & 120 & \\
\hline$\leq 12$ SNP cluster & & & & 0.016 \\
\hline Clustered & $36(58.1)$ & $40(38.5)$ & 76 & \\
\hline Not clustered & $26(41.9)$ & $64(61.5)$ & 90 & \\
\hline All patients & $62(100)$ & $104(100)$ & 166 & \\
\hline
\end{tabular}

*DST, drug susceptibility testing; L, lineage; SNP, single-nucleotide polymorphism. 
MDR TB cases were connected in 2 closely related molecular clusters (denoting recent transmission of MDR strains). This finding suggests that more focused infection control measures and contact tracing of patients with MDR TB need to be introduced to break the transmission chains at an early stage.

In this study, we found that a high proportion of culture-positive pulmonary TB cases $(73.4 \%)$ were

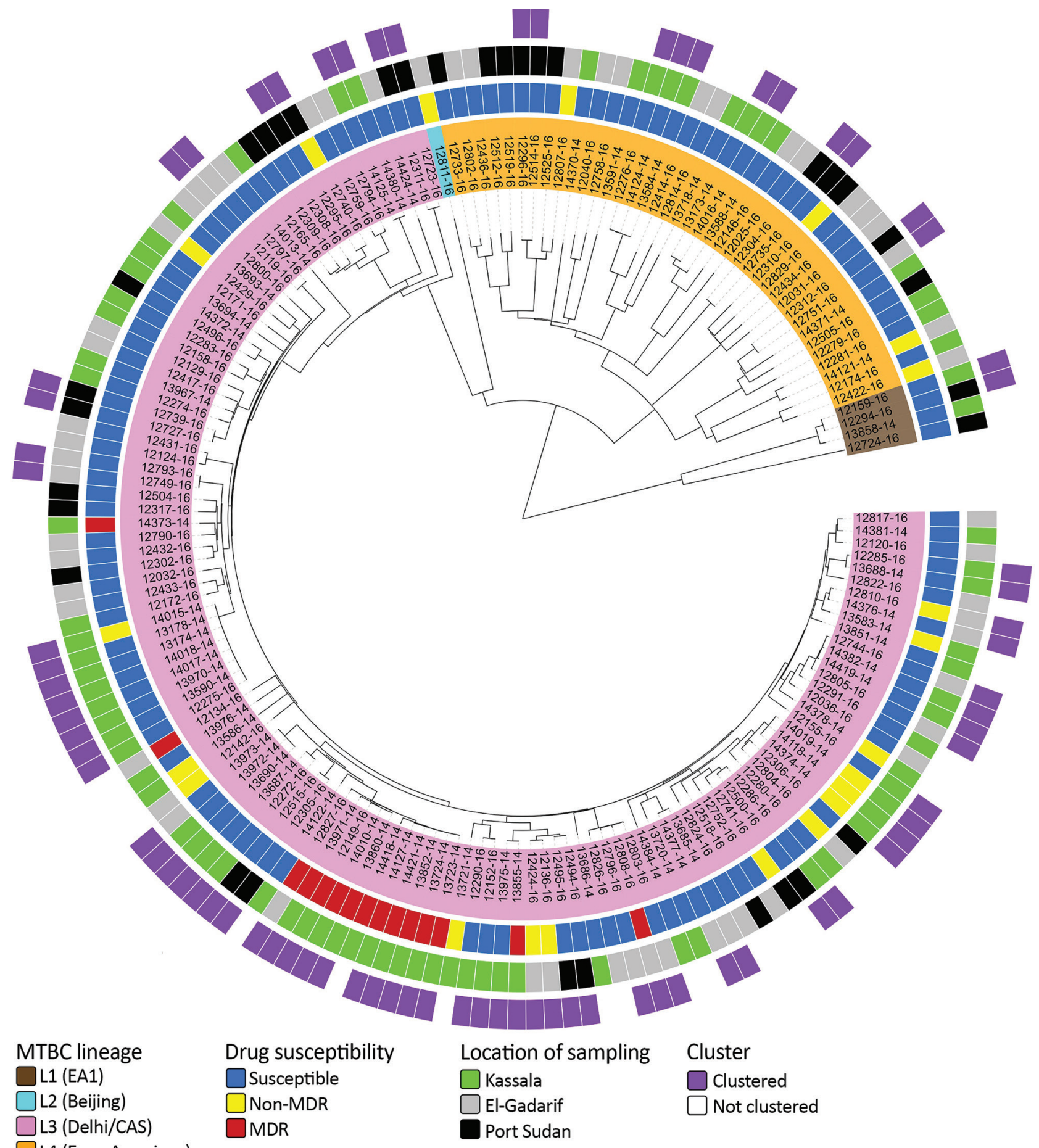

$\square$ L4 (Euro-American)

Figure 2. MTBC population structure in eastern Sudan. Maximum-likelihood tree based on 11,932 concatenated single-nucleotide polymorphisms (SNPs) using a general time-reversible substitution model. Colored bars code for (inner to outer ring) MTBC lineages (L1-4); genotypic DST results stratified to MDR, non-MDR, and pansusceptible; sampling location; and clustered and nonclustered strains (SNP distance $\leq 12$ ). MDR, multidrug resistant; MTBC, Mycobacterium tuberculosis complex. 
Table 3. Mutations conferring drug resistance among Mycobacterium tuberculosis complex genotypes identified in eastern Sudan, 2014 and $2016^{*}$

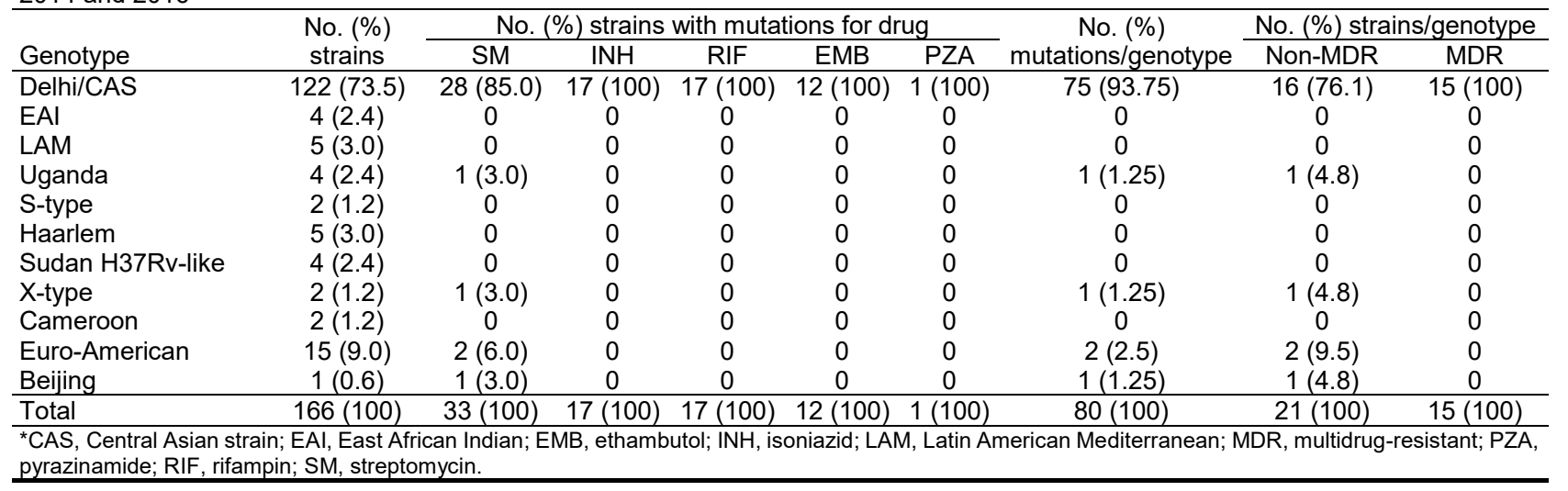

caused by L3 strains. This finding is in line with previous studies that have been based on classical genotyping methods and reported rates of $40 \%$ or higher of the so-called Central Asia spoligotype family in central and eastern Sudan $(5,15,35)$. Moreover, Couvin et al. (36) identified Sudan as an L3 hotspot in Africa. In this regard, it is intriguing to speculate whether L3 strains in general or certain subgroups have developed particular pathobiologic properties rendering them more virulent in East Africa host populations. Recently, Stucki et al. (37) hypothesized a concept of generalists and specialist among L4 strains based on the width of their geographic distribution. For L3 strains, larger studies are needed to reveal their global genetic diversity and geographic prevalence, which might inform about particular successful L3 subgroups and related causative variants in their genomes.

In addition to the general dominance of L3 strains in eastern Sudan, we found that all MDR TB cases were caused exclusively by L3 strains. Of major concern is that 10 of 15 MDR strains were part of 2 genetically related clusters isolated mainly from patients treated in 2014 in Kassala hospital. At first glance, this finding suggested nosocomial transmission. However, 2 strains of these clusters were isolated in 2016, including a strain from a patient treated in El-Gadarif hospital who had also acquired resistance to ethambutol and pyrazinamide. This patient was the first patient in our study cohort infected with a fully first-line drug-resistant strain, clearly emphasizing the importance of adopting focused TB control measures, including rapid detection and effective treatment of patients with MDR TB, to better contain transmission of MDR strains and prevent development of further drug resistances in the region. However, these measures are far from reality because proper TB diagnostics are virtually absent in eastern Sudan; other impeding factors are social stigma, lack of motivation, and poor awareness of TB treatment,

Table 4. Performance of genotypic drug resistance prediction to first-line tuberculosis drugs in Mycobacterium tuberculosis complex strains, eastern Sudan*

\begin{tabular}{|c|c|c|c|c|c|c|c|c|c|c|c|}
\hline \multirow[b]{2}{*}{ Drug } & \multicolumn{3}{|c|}{ Resistant } & \multicolumn{3}{|c|}{ Susceptible } & \multirow[b]{2}{*}{$\begin{array}{l}\text { Se, \%† } \\
(95 \% \mathrm{Cl})\end{array}$} & \multirow[b]{2}{*}{$\begin{array}{c}\text { Sp, \%‡ } \\
(95 \% \mathrm{Cl})\end{array}$} & \multirow[b]{2}{*}{$\begin{array}{l}\text { PPV, \%§ } \\
(95 \% \mathrm{Cl})\end{array}$} & \multirow[b]{2}{*}{$\begin{array}{l}\text { NPV, \% } \\
(95 \% \mathrm{Cl})\end{array}$} & \multirow{2}{*}{$\begin{array}{c}\text { Unknown } \\
\text { mutations } \\
(\%)\end{array}$} \\
\hline & $\begin{array}{l}\text { gR } \\
\text { (TP) }\end{array}$ & $\begin{array}{l}\mathrm{gS} \\
\text { (FN) }\end{array}$ & $\begin{array}{l}\mathrm{gU} \\
(\mathrm{FN})\end{array}$ & $\begin{array}{l}\mathrm{gR} \\
(\mathrm{FP})\end{array}$ & $\begin{array}{l}\mathrm{gS} \\
(\mathrm{TN})\end{array}$ & $\begin{array}{l}\mathrm{gU} \\
\text { (TN) }\end{array}$ & & & & & \\
\hline SM & 24 & 0 & 9 & 0 & 133 & 0 & $\begin{array}{c}72.7 \\
(54.5-86.7)\end{array}$ & 100 & 100 & $\begin{array}{c}93.7 \\
(89.4-96.3)\end{array}$ & $\begin{array}{c}9 / 166 \\
(5.4)\end{array}$ \\
\hline INH & 17 & 0 & 0 & 0 & 149 & 0 & 100 & 100 & 100 & 100 & $\begin{array}{l}0 / 166 \\
(0.0)\end{array}$ \\
\hline RIF & 17 & 0 & 0 & 0 & 149 & 0 & 100 & 100 & 100 & 100 & $\begin{array}{l}0 / 166 \\
(0.0)\end{array}$ \\
\hline EMB & 1 & 0 & 0 & $10^{*}$ & 155 & 0 & 100 & $\begin{array}{c}93.9 \\
(89.1-97.1)\end{array}$ & $\begin{array}{c}9.10 \\
(5.2-15.4)\end{array}$ & 100 & $\begin{array}{l}0 / 166 \\
(0.0)\end{array}$ \\
\hline PZA & 1 & 0 & 0 & 0 & 165 & 0 & 100 & 100 & 100 & 100 & $\begin{array}{c}0 / 166 \\
(0.0)\end{array}$ \\
\hline
\end{tabular}

*See MICs in the Methods section. EMB, ethambutol; FN, false negative; FP, false positive; gR, genetically resistant; gS, genetically susceptible; $\mathrm{gU}$, genetic resistance unknown; INH, isoniazid; NPV, negative predictive value; PPV, positive predictive value; PZA, pyrazinamide; RIF, rifampin; Se, sensitivity; Sp, specificity; SM, streptomycin; TN, true negative; TP, true positive.

$\mathrm{TSe}=\mathrm{TP} \div \mathrm{TP}+\mathrm{FN}$.

†Sp $=\mathrm{TN} \div \mathrm{TN}+\mathrm{FP}$.

SPPV $=T P \div(T P+F P)$.

TNPV $=\mathrm{TN} \div(\mathrm{TN}+\mathrm{FN})$. 
with default rates of $14 \%-57 \%(38,39)$. This situation may even lead to a further aggravation of the drug resistance problem through selection of MDR clones with additional drug resistances in failing treatment regimens and further transmission of fully first-line resistant MDR strains (14,39). However, our WGS analysis revealed that MDR isolates did not exhibit mutations mediating resistances to second-line drugs (except for isoniazid/ETH cross resistance), leaving reasonable therapeutic options for patients in eastern Sudan with MDR TB.

Former studies in central and eastern Sudan reported drug resistances in 39\%-67\% and MDR in $6 \%-22 \%$ of the strains investigated $(4-10)$. Those variations could possibly be attributed to dissimilarities in study design, sample size, and characteristics of study populations. In former studies, 53-235 samples from only new or new and retreatment TB cases with unknown HIV status or with a proportion of HIV-positive cases were investigated (4-10). Additionally, variations could also be linked to the laboratory technique used for pDST; for example, discordant results have been noticed between the BACTEC MGIT 960 and the proportion methods for streptomycin and ethambutol (40). In Sudan, only 1 study used the BACTEC MGIT 960; the remaining studies used the proportion method on LöwensteinJensen slants (4-10).

Considering the lack of pDST and the technical challenges associated with its implementation in Sudan, introduction of rapid molecular diagnostics to find patients with MDR TB is crucial for timely detection, treatment, and control. Moreover, rapid diagnostics will ultimately strengthen the national TB control program in Sudan. In line with previous studies, our data demonstrate an excellent performance of gDST for molecular resistance prediction $(16,17,41)$. One example of the benefits of molecular assays is the correction of false ethambutol susceptibility results based on pDST in strains that harbor high-confidence $e m b B$ resistance (42). Previous studies already revealed a low performance of ethambutol pDST, attributable mainly to the small difference between the wild-type and mutant MIC levels, leading to the effect that strains with canonical embB mutations show ethambutol MICs around the defined breakpoint of $5.0 \mu \mathrm{g} / \mathrm{mL}$, resulting in a low reproducibility of phenotypic results $(24,25,42)$. Therefore, classical Sanger sequencing of the $e m b B$ codons 306,354 , and 406 was recently proposed to overrule phenotypic ethambutol susceptibility results in cases of presence of mutations in these codons (42). Furthermore, Cepheid GeneXpert and Hain
MTBDRplus version 2.0 would have recognized all rifampin-resistant mediating mutations in our study setting and, therefore, offer a rapid solution for identification of patients with MDR/rifampin-resistant TB in eastern Sudan.

This multisite study was conducted in 3 public hospitals in eastern Sudan, comprising 10\%-20\% of the TB cases in the region during the study period; it thus represents a snapshot of the population diversity and transmission dynamics of MTBC strains in eastern Sudan. An additional strength of this study is that cultures, DSTs, and WGS were done in a World Health Organization-certified NRC in a highresource setting in Germany, enabling maximum resolution for characterization of MTBC strains.

This study had $\geq 2$ limitations. First, the prolonged transit time of patient-derived samples from Sudan to the NRC in Germany affected the viability of the MTBC bacteria; therefore, no mycobacterial growth was detected for some samples. Furthermore, the unavailability of clinical data, such as HIV status and treatment outcomes, prohibited further linking of bacteriological results to these clinical data.

In conclusion, L3 strains play a pivotal role in the epidemiology and transmission of $\mathrm{TB}$, particularly MDR TB, in eastern Sudan. Transmission of MDR TB could possibly be an emerging concern for local TB departments and hospitals. Therefore, to contain MDR TB transmission, rapid molecular diagnostics, such as Cepheid GeneXpert or Hain MTBDRplus v2.0, are desirable in combination with focused tracing of contacts of patients with MDR TB. In addition, early onset of MDR TB therapy would be an ideal approach to reduce the number of secondary cases.

\section{Acknowledgments}

We thank all the patients with TB who participated in this study as well as the physicians, technicians, and the working staff at the outpatient departments of public hospitals and local TB laboratories in eastern Sudan and at the Research Center Borstel in Germany for their assistance. In particular, we are grateful to I. Razio, P. Vock, B. Schlüter, T. Ubben, J. Zallet, K. Ott, A.-K. Witt, D. Sievert, and V. Mohr for providing excellent technical assistance. The contribution of the Ministry of Health in Kassala, in El-Gadarif, and in Port Sudan, eastern Sudan, and the Research Center Borstel, Borstel, Germany, in accomplishing this work is appreciated.

Y.A.S. is a DAAD (Deutscher Akademischer Austauschdienst or the German Academic Exchange Service) stipend recipient, funding program no. 57076385. Parts of this 
work have been supported by Deutsche Forschungsgemeinschaft (DFG, German Research Foundation) under Germany's Excellence Strategy EXC 22167-390884018, the Leibniz Science Campus EvoLUNG, and the German Center for Infection Research.

\section{About the Author}

Mr. Shuaib is a PhD student at the Research Center Borstel and Freie Universität Berlin in Germany and a teaching staff member at Sudan University of Science and Technology. He is interested in the epidemiology of infectious diseases, particularly zoonotic and transboundary animal diseases.

\section{References}

1. World Health Organization. Global tuberculosis report. 2019 Sep 26 [cited 2019 Oct 30]. https:/ / apps.who.int/iris/ bitstream/handle/10665/329368/9789241565714-eng.pdf

2. World Health Organization. Global tuberculosis report. 2012 Oct 17 [cited 2019 Mar 10]. https:/ / www.who.int/tb/ publications/global_report/gtbr12_main.pdf

3. Abdallah TM, Ali AA. Epidemiology of tuberculosis in eastern Sudan. Asian Pac J Trop Biomed. 2012;2:999-1001. https:/ / doi.org/10.1016/S2221-1691(13)60013-1

4. Muna OA. Determination of the prevalence of tuberculosis with drug-resistant strains of Mycobacterium tuberculosis in Khartoum, Gazira and camps for displaced people, Sudan. Oslo: Faculty of Medicine, University of Oslo; 2002.

5. Sharaf Eldin GS, Fadl-Elmula I, Ali MS, Ali AB, Salih AL, Mallard K, et al. Tuberculosis in Sudan: a study of Mycobacterium tuberculosis strain genotype and susceptibility to anti-tuberculosis drugs. BMC Infect Dis. 2011;11:219. https://doi.org/10.1186/1471-2334-11-219

6. Hassan SO, Musa MT, Elsheikh HM, Eleragi AM, Saeed OK. Drug resistance in Mycobacterium tuberculosis isolates from northeastern Sudan. Br J Med Res. 2012;2:424-33. https://doi.org/10.9734/BJMMR/2012/1404

7. Abdul-Aziz AA, Elhassan MM, Abdulsalam SA, Mohammed EO, Hamid ME. Multi-drug resistance tuberculosis (MDR-TB) in Kassala state, eastern Sudan. Trop Doct. 2013;43:66-70. https://doi.org/10.1177/ 0049475513490421

8. Khalid FA, Hamid ZA, Mukhtar MM. Tuberculosis drug resistance isolates from pulmonary tuberculosis patients, Kassala state, Sudan. Int J Mycobacteriol. 2015;4:44-7. https://doi.org/10.1016/j.ijmyco.2014.11.064

9. Nour EMM, Saeed EMA, Zaki AZSA, Saeed NS. Drug resistance patterns of Mycobacterium tuberculosis isolates from patients with pulmonary tuberculosis in the Sudan. IOSR Journal of Dental and Medical Sciences. 2015;14:17-9. https://doi.org/10.9790/0853-14881719

10. Eldirdery MM, Intisar EA, Mona OA, Fatima AK, Asrar MA, Nuha YI, et al. Prevalence of multidrug-resistant tuberculosis among smear positive pulmonary tuberculosis patients in eastern Sudan. Am J Microbiol Res. 2017;5:32-6. https:/ / doi.org/10.12691/ajmr-5-2-2

11. Auld SC, Kasmar AG, Dowdy DW, Mathema B, Gandhi NR, Churchyard GJ, et al. Research roadmap for tuberculosis transmission science: where do we go from here and how will we know when we're there? J Infect Dis. 2017;216(suppl_6):S662-8. https:/ / doi.org/10.1093/infdis/ jix353
12. Walker TM, Ip CL, Harrell RH, Evans JT, Kapatai G, Dedicoat MJ, et al. Whole-genome sequencing to delineate Mycobacterium tuberculosis outbreaks: a retrospective observational study. Lancet Infect Dis. 2013;13:137-46. https:/ / doi.org/10.1016/S1473-3099(12)70277-3

13. Merker M, Kohl TA, Niemann S, Supply P. The evolution of strain typing in the Mycobacterium tuberculosis complex. Adv Exp Med Biol. 2017;1019:43-78. https://doi.org/10.1007/978-3-319-64371-7_3

14. Shuaib YA, Khalil EAG, Schaible UE, Wieler LH, Bakheit MAM, Mohamed-Noor SE, et al. Smear microscopy for diagnosis of pulmonary tuberculosis in eastern Sudan. Tuberc Res Treat. 2018;2018:8038137. https://doi.org/10.1155/2018/8038137

15. Khalid FA, Gasmelseed N, Hailu E, Eldirdery MM, Abebe M, Berg S, et al. Molecular identification of Mycobacterium tuberculosis causing pulmonary tuberculosis in Sudan. Eur Acad Res. 2016;4:7842-55.

16. Gröschel MI, Walker TM, van der Werf TS, Lange C, Niemann S, Merker M. Pathogen-based precision medicine for drug-resistant tuberculosis. PLoS Pathog. 2018; 14:e1007297. https:/ / doi.org/10.1371/journal.ppat.1007297

17. Meehan CJ, Goig GA, Kohl TA, Verboven L, Dippenaar A, Ezewudo M, et al. Whole genome sequencing of Mycobacterium tuberculosis: current standards and open issues. Nat Rev Microbiol. 2019;17:533-45. https:/ / doi.org/ 10.1038/s41579-019-0214-5

18. Siddiqi S, Rüsch-Gerdes S. MGIT TM Procedure Manual. Geneva: Foundation for Innovative New Diagnostics (FIND); 2006.

19. Deutsches Institut für Normung. Medical microbiologydiagnosis of tuberculosis. Part 3: detection of mycobacteria by culture methods. Berlin: The Institute; 2011.

20. Somerville W, Thibert L, Schwartzman K, Behr MA. Extraction of Mycobacterium tuberculosis DNA: a question of containment. J Clin Microbiol. 2005;43:2996-7. https://doi.org/10.1128/JCM.43.6.2996-2997.2005

21. Hillemann D, Warren R, Kubica T, Rüsch-Gerdes S, Niemann S. Rapid detection of Mycobacterium tuberculosis Beijing genotype strains by real-time PCR. J Clin Microbiol. 2006;44:302-6. https:// doi.org/10.1128/JCM.44.2. 302-306.2006

22. Richter E, Niemann S, Gloeckner FO, Pfyffer GE, Rüsch-Gerdes S. Mycobacterium holsaticum sp. nov. Int J Syst Evol Microbiol. 2002;52:1991-6.

23. Rüsch-Gerdes S, Pfyffer GE, Casal M, Chadwick M, Siddiqi S. Multicenter laboratory validation of the BACTEC MGIT 960 technique for testing susceptibilities of Mycobacterium tuberculosis to classical second-line drugs and newer antimicrobials. J Clin Microbiol. 2006;44:688-92. https:// doi.org/10.1128/JCM.44.3.688-692.2006

24. Ängeby K, Juréen P, Kahlmeter G, Hoffner SE, Schön T. Challenging a dogma: antimicrobial susceptibility testing breakpoints for Mycobacterium tuberculosis. Bull World Health Organ. 2012;90:693-8. https:/ / doi.org/10.2471/ BLT.11.096644

25. Heyckendorf J, Andres S, Köser CU, Olaru ID, Schön T, Sturegård E, et al. What is resistance? Impact of phenotypic versus molecular drug resistance testing on therapy for multi- and extensively drug-resistant tuberculosis. Antimicrob Agents Chemother. 2018;62:e01550-17. https:/ / doi.org/doi: 10.1128/AAC.01550-17

26. Merker M, Barbier M, Cox H, Rasigade JP, Feuerriegel S, Kohl TA, et al. Compensatory evolution drives multidrugresistant tuberculosis in Central Asia. eLife. 2018;7:e38200. https://doi.org/10.7554/eLife.38200 
27. Kohl TA, Utpatel C, Schleusener V, De Filippo MR, Beckert P, Cirillo DM, et al. MTBseq: a comprehensive pipeline for whole genome sequence analysis of Mycobacterium tuberculosis complex isolates. PeerJ. 2018; 6:e5895. https://doi.org/10.7717/peerj.5895

28. Price MN, Dehal PS, Arkin AP. FastTree 2-approximately maximum-likelihood trees for large alignments. PLoS One. 2010;5:e9490. https://doi.org/10.1371/journal.pone.0009490

29. He Z, Zhang H, Gao S, Lercher MJ, Chen WH, Hu S. Evolview v2: an online visualization and management tool for customized and annotated phylogenetic trees. Nucleic Acids Res. 2016;44:W236-41. https:/ / doi.org/10.1093/nar/ gkw370

30. Kannan L, Wheeler WC. Maximum parsimony on phylogenetic networks. Algorithms Mol Biol. 2012;7:9. https:/ / doi.org/10.1186/1748-7188-7-9

31. Feuerriegel S, Schleusener V, Beckert P, Kohl TA, Miotto P, Cirillo DM, et al. PhyResSE: a Web tool delineating Mycobacterium tuberculosis antibiotic resistance and lineage from whole-genome sequencing data. J Clin Microbiol. 2015;53:1908-14. https://doi.org/10.1128/JCM.00025-15

32. Kim HY. Statistical notes for clinical researchers: chi-squared test and Fisher's exact test. Restor Dent Endod. 2017; 42:152-5. https:// doi.org/10.5395/rde.2017.42.2.152

33. Coll F, McNerney R, Guerra-Assunção JA, Glynn JR, Perdigão J, Viveiros M, et al. A robust SNP barcode for typing Mycobacterium tuberculosis complex strains. Nat Commun. 2014;5:4812. https://doi.org/10.1038/ncomms5812

34. Meehan CJ, Moris P, Kohl TA, Pečerska J, Akter S, Merker M, et al. The relationship between transmission time and clustering methods in Mycobacterium tuberculosis epidemiology. EBioMedicine. 2018;37:410-6. https:/ / doi.org/ 10.1016/j.ebiom.2018.10.013

35. Eldirdery MM, Alrayah IE, ElkareIm MOA, Khalid FA, Elegail AMA, Ibrahim NY, et al. Genotyping of pulmonary Mycobacterium tuberculosis isolates from Sudan using spoligotyping. Am J Microbiol Res. 2015;3:125-8.

36. Couvin D, Reynaud Y, Rastogi N. Two tales: worldwide distribution of Central Asian (CAS) versus ancestral
East-African Indian (EAI) lineages of Mycobacterium tuberculosis underlines a remarkable cleavage for phylogeographical, epidemiological and demographical characteristics. PLoS One. 2019;14:e0219706. https:/ / doi.org/ 10.1371/journal.pone.0219706

37. Stucki D, Brites D, Jeljeli L, Coscolla M, Liu Q, Trauner A, et al. Mycobacterium tuberculosis lineage 4 comprises globally distributed and geographically restricted sublineages. Nat Genet. 2016;48:1535-43. https:// doi.org/10.1038/ng.3704

38. Abu Shanab ME. Defaulting to anti-tuberculosis treatment: proportional and associated factors among internally displaced people around Khartoum state. Khartoum (Sudan): Faculty of Public and Environmental Health, University of Khartoum; 2003.

39. Ali AO, Prins MH. Patient non adherence to tuberculosis treatment in Sudan: socio demographic factors influencing non adherence to tuberculosis therapy in Khartoum state. Pan Afr Med J. 2016;25:80. https://doi.org/10.11604/ pamj.2016.25.80.9447

40. Giampaglia CM, Martins MC, Vieira GB, Vinhas SA, Telles MA, Palaci M, et al. Multicentre evaluation of an automated BACTEC 960 system for susceptibility testing of Mycobacterium tuberculosis. Int J Tuberc Lung Dis. 2007;11:986-91.

41. CRyPTIC Consortium and the 100,000 Genomes Project. Prediction of susceptibility to first-line tuberculosis drugs by DNA sequencing. N Engl J Med. 2018;379:1403-15. https:/ / doi.org/10.1056/NEJMoa1800474

42. Andres S, Gröschel MI, Hillemann D, Merker M,

Niemann S, Kranzer K. A diagnostic algorithm to investigate pyrazinamide and ethambutol resistance in rifampinresistant Mycobacterium tuberculosis isolates in a lowincidence setting. Antimicrob Agents Chemother. 2019;63:e01798-18. https:/ / doi.org/10.1128/AAC.01798-18

Address for correspondence: Yassir A. Shuaib or Matthias Merker, Research Center Borstel, Parkallee 1-40, 23845 Borstel, Germany; email: vet.aboamar@gmail.com or mmerker@fz-borstel.de

\section{etymologia}

Etymology is concerned with the origin of words, how they ve evolved over time, and changed in form and meaning as they were translated from one language to another. Every month, EID publishes a feature highlighting the etymology of a word from medicine or public health.

\section{featured monthy in EMERGING} http://wwwnc.cdc.gov/eid/articles/etymologia INFECTIOUS DISEASES 